\title{
Preliminary optical design for the common fore optics of METIS
}

\author{
Tibor Agócs ${ }^{\mathrm{a}}$, Bernhard R. Brandl ${ }^{\mathrm{b}}$, Rieks Jager ${ }^{\mathrm{a}}$, Felix Bettonvil ${ }^{\mathrm{a}}$, Gabby Aitink-Kroes ${ }^{\mathrm{a}}$, Lars \\ Venema $^{\mathrm{c}}$, Matthew Kenworthy ${ }^{\mathrm{b}}$, Olivier Absil ${ }^{\mathrm{d}}$, Thomas Bertram ${ }^{\mathrm{e}}$

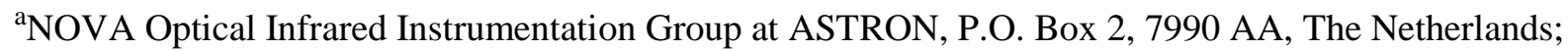 \\ ${ }^{\mathrm{b}}$ Leiden Observatory, Leiden University, P.O. Box 9513, 2300 RA Leiden, The Netherlands; ${ }^{\mathrm{c} A S T R O N, ~ P . O . ~}$ \\ Box 2, 7990 AA Dwingeloo, The Netherlands; 'Département d'Astrophysique, Géophysique et \\ Océanographie, Université de Liège, Allée du Six Août 17, 4000 Liège, Belgium, ${ }^{\mathrm{e}}$ Max Planck Institut für \\ Astronomie, Königstuhl 17, D-69117, Heidelberg, Germany
}

\begin{abstract}
METIS is the Mid-infrared E-ELT Imager and Spectrograph, which will provide outstanding observing capabilities, focusing on high angular and spectral resolution. It consists of two diffraction-limited imagers operating in the LM and NQ bands respectively and an IFU fed diffraction-limited high-resolution $(\mathrm{R}=100,000) \mathrm{LM}$ band spectrograph. These science subsystems are preceded by the common fore optics (CFO), which provides the following essential functionalities: calibration, chopping, image de-rotation, thermal background and stray light reduction. We show the evolution of the CFO optical design from the conceptual design to the preliminary optical design, detail the optimization steps and discuss the necessary trade-offs.
\end{abstract}

Keywords: METIS, E-ELT, mid-infrared, fore optics, preliminary design, re-imager, Offner-relay, system optimization

\section{INTRODUCTION}

METIS is the Mid-infrared E-ELT Imager and Spectrograph, which will provide outstanding observing capabilities, focusing on high angular and spectral resolution on the largest telescope on Earth. The optical overview of METIS is shown in Figure 1. It consists of two diffraction-limited imagers (IMG), providing also low and medium resolution long slit spectroscopy, operating in the LM and NQ bands respectively and an IFU fed diffraction-limited high-resolution $(\mathrm{R}=100,000) \mathrm{LM}$ band spectrograph (LMS). These science subsystems are preceded by the common fore optics (CFO), which re-images the focal plane of the EELT inside METIS and provides the following essential functionalities: calibration, chopping, image de-rotation, thermal background and stray light reduction. The CFO will be the core optical system of the instrument and it will provide the interface towards the imagers, the spectrograph, the single conjugate adaptive optics system (SCA) and the warm calibration unit (WCU). METIS will also provide coronagraphy for all operating modes to achieve high contrast imaging and the focal and pupil planes of the CFO will be populated by the components of apodizing phase plate and vortex coronagraphs. More information on the project overview and science cases can be found in the paper by Brandl et al. [1].

As a starting point, in section 2, we discuss the high level requirements and detail the most critical ones that have the largest influence on the optical design of the CFO. In section 0 , we present the numerical scoring method that we use in the trade-off analysis, which aims to determine the order of key components in the CFO that best fulfils the relevant scientific, operational and technical requirements. In section 4 we show different spherical and aspherical optical architectures that were considered in the course of the optical design process since the start of phase B. In section 5 we present the proposed optical design for the CFO, which is based on an all-spherical system and consists of a double reimager: a classical and a re-optimized Offner relay. We present the optical characteristics of the focal and the pupil planes, detail the manufacturing strategy of the optical components and discuss the preliminary AIV plan. We elaborate on folding and packaging that are always essential parts of the opto-mechanical design process, especially for cryogenic instruments. In section 6 , we summarize the achievements and outline the future challenges of the optical design process of the CFO.

* agocs@ astron.nl; Tel +31 521595173

Ground-based and Airborne Instrumentation for Astronomy VI, edited by Christopher J. Evans, Luc Simard, Hideki Takami Proc. of SPIE Vol. 9908, 99089Q - (c) 2016 SPIE · CCC code: 0277-786X/16/\$18 · doi: 10.1117/12.2232512 


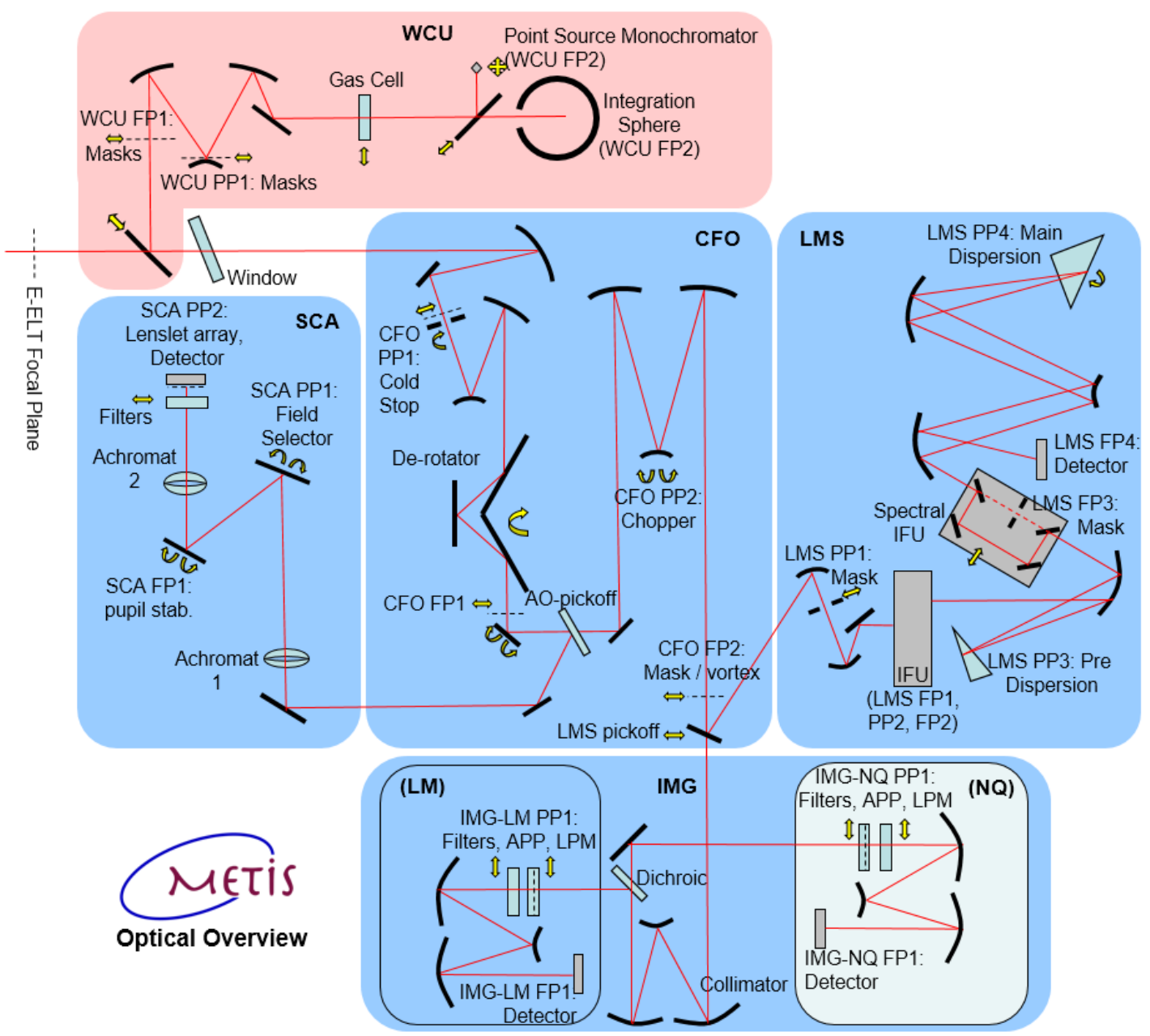

Figure 1 The optical overview of METIS is shown.

\section{REQUIREMENTS}

In the following the most critical requirements are presented from the perspective of the CFO and more details are given on how they are driving the optical design.

\subsection{LTAO-METIS interface requirement}

The interface between the LTAO system and METIS is a plane that is perpendicular to the optical axis and $750 \mathrm{~mm}$ downstream from the EELT focal plane. METIS shall not extend beyond this interface. In the proposed baseline optical design the WCU feed mirror can be inserted in the space between $750 \mathrm{~mm}-1000 \mathrm{~mm}$ and the cryostat window is $1000 \mathrm{~mm}$ downstream from the EELT focal plane. In phase A this distance was significantly shorter, $500 \mathrm{~mm}$. The deviation from phase A drives the optical design in terms of increased optical path length, more components, more complexity and especially difficulty in designing a suitable pupil for the chopper with phase A specifications (size, shape and chop-throw parameters).

\subsection{Requirement of having a double reimager}

The requirement to have a double reimager in the CFO can be derived from several high level requirements. The CFO shall provide a co-rotating cold stop in transmission to limit thermal radiation. Since the reflective chopper also have to be in the pupil, it means that a second, accessible pupil is needed in transmission. Pupil stabilization is not decided yet, nevertheless it can only be accommodated by a double reimager design in the CFO. Coronagraphy related requirements 
also require double reimager in the CFO: final coronagraphic modes are not decided yet either, but coronagraphy using Ring Apodized Vortex Coronagraph (RAVC) for IMG and coronagraphy for LMS are the main drivers here (see also the paper by Kenworthy et al [2]). Stray light control can also be done very efficiently in a double reimager design (cold stop in transmission, more location for baffling and vanes, field stops, etc.). Finally, one of the main drivers of the double reimager design is that by providing a longer optical path, additional focal and pupil planes, a better optimization can be done regarding the order of key components that leads to a better overall optical design (see more details on this in section $0)$.

\subsection{Field of view and sampling requirements}

The FOV of the CFO is driven by the FOV and chopping necessary for the IMG subsystem. The FOV of the imager is driven by the sampling requirement of the LM and NQ band imagers and the pixel sizes of the detectors. The FOV is 10.5" x 10.5" for LM (2000x2000 pixels, 5.25 mas pixels) and 15.0" x 15.0" for NQ (1000x1000 pixels, 15.0 mas pixels); some edge pixels are left for calibration purposes. The larger 15.0" x 15.0" FOV for the NQ band imager is driving and with the additional 10" chopping ( 5 " chopping is necessary in all direction) plus $\sim 1$ " margin the complete CFO FOV sums up to 33" diameter. The CFO shall provide 33" circular FOV up to the chopper and after the chopper the FOV is reduced to a 23 " circular FOV. As in any optical system, the larger the FOV, the more difficult the optical design is, and another challenging requirement is that the image quality of the full chopped FOV should be excellent (it shall not decrease nonchopped RMS WFE by more than 5\%).

\subsection{Cold stop requirements}

All cold stop requirements are related to the need of efficient background handling in the instrument. A cold stop that corotates with the entrance pupil of the EELT and covers the spiders of the telescope can achieve better background reduction and also offers the possibility to use non-circular geometrical shape for the cold stop and achieve higher throughput. Additionally, if the cold stop is in transmission and not in reflection (phase A situation), the stray light characteristics of the design are much better, since all the light that is reflected back from the cold stop propagate backwards in the optical system. Other requirements are connected to the dimensioning of the cold stop with respect to the entrance pupil of the EELT: the cold stop diameter shall be undersized with respect to the 'all-glass' pupil of the EELT, its central obscuration and spiders shall be oversized with respect to the 'all- glass' central obscuration and spiders of the EELT. In Table 1 the pupil budget is shown, which drives the dimensioning of the cold stop with respect to the mentioned all-glass dimensions. In the table, $D$ is the nominal entrance pupil diameter of the EELT conjugated at the cold stop pupil.

Table 1 Pupil budget for cold stop dimensioning. $\mathrm{D}$ is the nominal entrance pupil diameter of the EELT conjugated at the cold stop pupil. Linear sum is used for the calculation of the total error to address the worst case scenario and not allow any thermal leak from outside the M1 segments.

\begin{tabular}{|l|c|}
\hline Source of error & Maximum allowed \\
\hline $\begin{array}{l}\text { Static pupil errors: pupil shear, distortion and elongation for the full FOV between } \\
\text { EELT entrance pupil and cold stop. }\end{array}$ & $0.015 * \mathrm{D}$ \\
\hline $\begin{array}{l}\text { Pupil alignment residuals (alignment residual between the EELT and METIS), both } \\
\text { decentre x/y and misalignment along the optical axis }\end{array}$ & $0.002 * \mathrm{D}$ \\
\hline $\begin{array}{l}\text { Pupil stability of the EELT } \\
\text { During operation, the exit pupil lateral position shall be stable to within }+/-0.5 \% \\
\text { (TBC) of the pupil diameter per axis over a period of 1 hour. }\end{array}$ & $0.005 * \mathrm{D}$ \\
\hline Total pupil errors & $0.022 * \mathrm{D}$ \\
\hline Safety margin & $0.003 * \mathrm{D}$ \\
\hline Total pupil errors with safety margin & $\mathbf{0 . 0 2 5}$ D \\
\hline
\end{tabular}

As a result of the co-rotating cold stop, a non-circular geometrical shape can also be considered. Following the 'natural' shape of the EELT M1, namely a hexagon for the central obscuration and a 12 sided polygon for the outside, one can compare the possible gain in throughput. In Figure 2 the circular and non-circular (hexagonal-12 sided polygon) cold stop shapes are shown in overlay with the entrance pupil of the EELT. Considering the $2.5 \%$ oversize that was determined from the budget presented above and keeping the same requirement for the dimensioning of the two cold stop pupil shapes, it can be calculated that the area of the hexagonal-12 sided polygon cold stop is 5.6\% larger than the circular cold stop. The pupil budget presented above corresponds to the pupil errors between the EELT entrance pupil and the cold stop pupil. When the cold stop is located in the first pupil of the CFO, it is necessary to control the maximum pupil elongation and shear for the subsequent pupils to limit transmission loss, when using pupil masks for certain HCI modes. Therefore the 
additional requirement is that the pupil elongation and shear should be smaller than 3\% (TBD) between the cold stop and the subsequent pupil in the CFO.
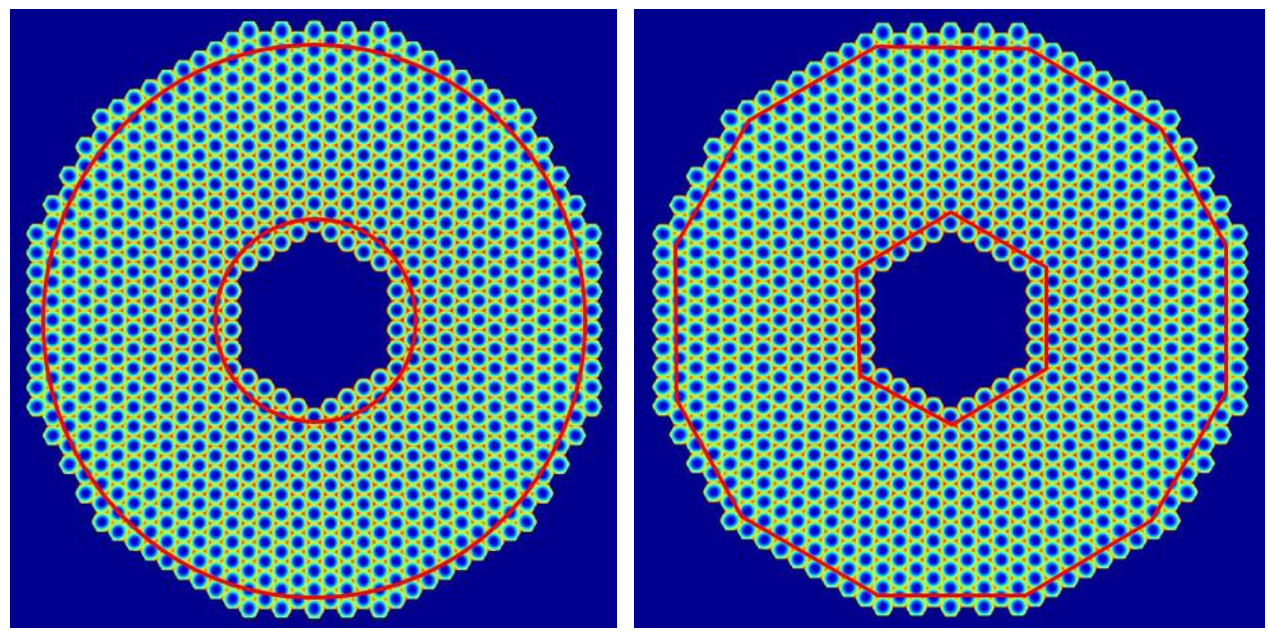

Figure 2 Possible cold stop shapes as projected over the M1 of the EELT. Left circular shape, right hexagonal for the central obscuration, 12 sided polygon for the outside.

\subsection{Coronagraphy requirements}

In Table 2 the complete list of High Contrast Imaging (HCI) modes considered. Two major coronagraphy systems are indicated: Apodizing Phase Plate (APP) coronagraphs and Vortex Coronagraphs (VC). The latter has three different flavors. 'Classical VC' is a classical vortex coronagraph, with an Annular Groove Phase Mask (AGPM) at the focus and a standard Lyot Stop (LS). The 'LPM-VC' is a vortex coronagraph with a Lyot-plane Phase Mask (an APP-like device, which replaces the Lyot stop). The 'RAVC' is a ring-apodized vortex coronagraph, which has an upstream amplitude apodizer and a downstream Lyot stop or LPM.

The requirement of having a double reimager in the CFO is driven by RAVC for IMG and coronagraphy for LMS. Additionally, for the vortex type coronagraph the image stability in the focal plane, where the AGPM is located is essential. Typically, a very demanding $0.01 \lambda / \mathrm{D}$ is necessary and so the careful definition of field stabilization strategy using the chopper and M5 of the EELT is necessary. Apart from the jitter requirement, pupil quality in the instrument is also crucial for the coronagraphic modes. Pupil shear, distortion, elongation and pupil stability affects coronagraphic modes and similarly to the strategy of background reduction, under-sizing of the coronagraphic masks are necessary, which in turn affects coronagraphic performance. For all coronagraphic modes pupil tracking is necessary and as a general rule, as few moving components should be in the optical path as possible.

Table 2 The complete list of coronagraphy (or HCI) modes considered. FP means Focal Plane, PP means Pupil Plane.

\begin{tabular}{|c|c|c|c|c|c|c|}
\hline Name of HCI mode & HCI for & CFO FP1 & CFO PP2 & CFO FP2 & IMG PP & LMS-SPO PP \\
\hline APP-1 ALL & IMG/ LMS & & APP & & & \\
\hline APP-1 LM/NQ IMG & IMG & & & & APP & \\
\hline APP-1 LMS & LMS & & & & & APP \\
\hline Classical/LPM-VC LM/NQ IMG v1 & IMG & & & AGPM & LS/LPM & \\
\hline Classical/LPM-VC LM/NQ IMG v2* & IMG & AGPM & & & $\mathrm{LS} / \mathrm{LPM}$ & \\
\hline Classical/LPM-VC LMS v1* & LMS & AGPM & & & & LS/LPM \\
\hline Classical/LPM-VC LMS v2* & IMG/ LMS & AGPM & LS/LPM & & & \\
\hline RAVC LM/NQ IMG & IMG & & Ring-Apod & AGPM & LS/LPM & \\
\hline
\end{tabular}

* These modes are not possible with the proposed CFO optical design. 


\section{TRADE-OFF ON THE ORDER OF KEY COMPONENTS}

A trade-off study was made to investigate the order of key components in the double reimager based CFO. The key components with the acronyms that were used in this study are the following: $A O$ : AO-pickoff, $D E$ : derotator, $C H$ : chopper, $C O$ : co-rotating cold stop and IF: intermediate focal plane (also referred to as FP1). A scoring scheme was set up to determine the best configuration: relevant requirements/goals were identified and different rules (hard and soft) were defined based on design, technological, science and operation constraints. Hard rules have to be always satisfied, soft rules have a certain benefit for the instrument and they have weights (ranging between 0.5 and 3.0 in 0.5 steps) indicating their importance and impact (Table 3).

Table 3 The hard and soft rules that are used to determine the best order of key components in the CFO are listed in the table.

$\mathrm{H} 1, \mathrm{H} 2$ are hard rules that have to be satisfied always.

\begin{tabular}{|c|c|c|c|c|}
\hline \multirow{2}{*}{ Num } & \multirow{2}{*}{ Description } & \multirow{2}{*}{ Component order } & \multicolumn{2}{|c|}{ Weight } \\
\hline & & & $\mathrm{V} 1$ & $\mathrm{~V} 2$ \\
\hline H1 & $\begin{array}{l}\text { Chopper shall be after the AO pickoff (otherwise counter chopping is necessary in } \\
\text { SCA). }\end{array}$ & $\mathrm{AO}$ then $\mathrm{CH}$ & $\infty$ & $\infty$ \\
\hline $\mathrm{H} 2$ & Derotator can not be before both pupils, due to lack of space. & DE can not be first & $\infty$ & $\infty$ \\
\hline $\mathrm{S} 1$ & $\begin{array}{l}\text { AO pickoff should be as far downstream in the optical path as possible to reduce } \\
\text { NCPA-s (to improve SCA performance). }\end{array}$ & $\begin{array}{l}\mathrm{AO} \text { is in } 4 \text { th, } 5 \text { th } \\
\text { place }\end{array}$ & 1 & 1,5 \\
\hline S2 & $\begin{array}{l}\text { AO pickoff should be after the derotator. To achieve better derotator tolerances. } \\
\text { To avoid focal plane drift when SCA uses off-axis target. To achieve field rotation } \\
\text { in SCA (field selector doesn't need field rotation). }\end{array}$ & $\mathrm{DE}$ then $\mathrm{AO}$ & 1 & 2,5 \\
\hline S3 & $\begin{array}{l}\text { Chopper should be in PP1 (beam is smaller afterwards, meaning smaller optics, } \\
\text { smaller derotator, smaller FOV). }\end{array}$ & $\mathrm{CH}$ then $\mathrm{CO}$ & 1 & 1 \\
\hline S4 & $\begin{array}{l}\text { Derotator should be after PP2 (two reimagers are compensating pupil elongation } \\
\text { and shear, which means smaller cold stop undersize and larger throughput). }\end{array}$ & $\begin{array}{l}\mathrm{CH} \text { then } \mathrm{DE} \text { or } \mathrm{CO} \\
\text { then } \mathrm{DE}\end{array}$ & 1 & 1 \\
\hline S5 & $\begin{array}{l}\text { IF (FP1) should be after the chopper to compensate low frequency (residual) image } \\
\text { jitter. It is for AGPM at FP1 only and there is a solution: AGPM + LMS pickoff } \\
\text { in FP2. }\end{array}$ & $\mathrm{CH}$ then IF & 1 & 0 \\
\hline S6 & $\begin{array}{l}\text { IF (FP1) should be after the chopper. Use of the chopper for small image offsets } \\
\text { within the IFU field. It is for AGPM at FP1 only and there is a solution: AGPM + } \\
\text { LMS pickoff in FP2. }\end{array}$ & $\mathrm{CH}$ then IF & 1 & 0 \\
\hline S7 & $\begin{array}{l}\text { IF (FP1) should be after the AO dichroic. Otherwise object behind the AGPM can } \\
\text { not be used for AO. It is for AGPM at FP1 only and there is a (not perfect) solution: } \\
\text { AGPM + LMS pickoff in FP2. }\end{array}$ & AO then IF & 1 & 1 \\
\hline S8 & $\begin{array}{l}\text { The AO pickoff should not be between the derotator and the intermediate focal } \\
\text { plane, because derotator size increases significantly. }\end{array}$ & $\begin{array}{l}\text { No DE-AO-IF or no } \\
\text { IF-AO-DE }\end{array}$ & 1 & 3 \\
\hline S9 & $\begin{array}{l}\text { AO pickoff should be close to the intermediate or output focal plane (to minimize } \\
\text { size). }\end{array}$ & $\begin{array}{l}\mathrm{AO}-\mathrm{IF} \text { or } \mathrm{IF}-\mathrm{AO} \\
\text { exact order }\end{array}$ & 1 & 0,5 \\
\hline S10 & $\begin{array}{l}\text { Pupil stabilization should be before the cold stop to maximize cold stop size and } \\
\text { throughput. }\end{array}$ & IF then $\mathrm{CO}$ & 1 & 0,5 \\
\hline S11 & $\begin{array}{l}\text { Coronagraph pupil (same as } \mathrm{CO} \text { ) should be after the AO pickoff. Otherwise in this } \\
\text { pupil APP can not be used (affects AO). }\end{array}$ & $\mathrm{AO}$ then $\mathrm{CO}$ & 1 & 1 \\
\hline $\mathrm{S} 12$ & $\begin{array}{l}\text { Pupil stabilization should be before the AO pickoff (closed loop pupil } \\
\text { stabilization). }\end{array}$ & IF then $\mathrm{AO}$ & 1 & 0,5 \\
\hline S13 & $\begin{array}{l}\text { Cold stop should be after the AO-pickoff. Otherwise it is difficult to determine } \\
\text { pupil shift with the SCA. }\end{array}$ & $\mathrm{AO}$ then $\mathrm{CO}$ & 1 & 0,5 \\
\hline S14 & $\begin{array}{l}\text { Derotator should be as far as upstream as possible to reduce the amount of rotating } \\
\text { speckles in pupil tracking mode (for all HCI). }\end{array}$ & $\begin{array}{l}\text { DE is in } 2 \text { nd or } 3 \text { rd } \\
\text { place }\end{array}$ & 1 & 0,5 \\
\hline S15 & $\begin{array}{l}\text { AO pickoff is as close as possible to coronagraphic devices (AGPM, APP) to } \\
\text { reduce NCPA-s. }\end{array}$ & $\begin{array}{l}\text { AO-IF, IF-AO, AO- } \\
\mathrm{CO}, \mathrm{CO}-\mathrm{AO}\end{array}$ & 1 & 0,5 \\
\hline
\end{tabular}


Since there are too many requirements/goals that influence the order of the components in the CFO, the scoring scheme provided a simple way to determine the best configuration. It was also easy to update and manage the requirements immediately see their effect on certain configurations.

In Table 4 all the configurations that satisfy the hard rules were listed and their scores were calculated ( 0 or 1 points were given to them and then multiplied by the weights) for two scenarios: v1 solely indicates the number of soft rules satisfied and v2 also contains the weights considered. Naturally, the rules listed above take into account the lessons learnt in the optical design process that was done in parallel with this activity. The weights were adjusted so that each two rules were weighted against each other and the relative difficulty, complexity and risk are reflected in the weights given to them. The highest ranked configuration is highlighted green in the table. It represents the following order of key components: cold stop, derotator, intermediate focal plane, AO pick-off and chopper. Compared to alternative configurations, the following are the main advantages. Since the AO-pickoff is further downstream, it has less NCPA between SCA and science focal planes (IMG and LMS). Additionally, the AO-pickoff is smaller in size (the long axis is $180 \mathrm{~mm}$ ), which is beneficial for the manufacturing of the blank and the dichroic coating. The main benefit is that there is a common derotator for SCA and the science channel (IMG, LMS). Firstly, it means that the stringent requirements on the derotator (manufacturing, alignment, stability) could be loosened due to the closed loop operation of the SCA system. Secondly, focal plane drift is not present, when SCA uses off-axis target (which is a major issue for some alternative designs), especially for long slit spectroscopy modes. Thirdly, field rotation can be done in SCA, so the field selector doesn't need to provide field rotation.

Table 4 The scores of all configurations are listed and the highest ranked configuration is highlighted green.

\begin{tabular}{|c|c|c|c|c|c|c|c|c|c|c|c|c|c|c|c|c|c|}
\hline \multirow{2}{*}{ Order } & \multicolumn{2}{|c|}{ Score } & \multirow{2}{*}{ S1 } & \multirow{2}{*}{$\mathbf{S 2}$} & \multirow{2}{*}{ S3 } & \multirow{2}{*}{ S4 } & \multirow{2}{*}{ S5 } & \multirow{2}{*}{ S6 } & \multirow{2}{*}{ S7 } & \multirow{2}{*}{ S8 } & \multirow{2}{*}{ S9 } & \multirow{2}{*}{ S10 } & \multirow{2}{*}{ S11 } & \multirow{2}{*}{ S12 } & \multirow{2}{*}{ S13 } & \multirow{2}{*}{ S14 } & \multirow{2}{*}{ S15 } \\
\hline & V1 & V2 & & & & & & & & & & & & & & & \\
\hline $\begin{array}{l}\text { CO-IF-AO- } \\
\text { DE-CH }\end{array}$ & 3 & 1,5 & 0 & 0 & 0 & 0 & 0 & 0 & 0 & 0 & 1 & 0 & 0 & 1 & 0 & 0 & 1 \\
\hline $\begin{array}{l}\text { CO-AO-IF- } \\
\text { DE-CH }\end{array}$ & 4 & 5 & 0 & 0 & 0 & 0 & 0 & 0 & 1 & 1 & 1 & 0 & 0 & 0 & 0 & 0 & 1 \\
\hline $\begin{array}{l}\text { CO-AO-DE- } \\
\text { IF-CH }\end{array}$ & 4 & 5 & 0 & 0 & 0 & 0 & 0 & 0 & 1 & 1 & 0 & 0 & 0 & 0 & 0 & 1 & 1 \\
\hline $\begin{array}{l}\text { CO-IF-AO- } \\
\text { CH-DE }\end{array}$ & 5 & 5,5 & 0 & 0 & 0 & 1 & 0 & 0 & 0 & 1 & 1 & 0 & 0 & 1 & 0 & 0 & 1 \\
\hline $\begin{array}{l}\text { CO-AO-IF- } \\
\text { CH-DE }\end{array}$ & 5 & 6 & 0 & 0 & 0 & 1 & 0 & 0 & 1 & 1 & 1 & 0 & 0 & 0 & 0 & 0 & 1 \\
\hline $\begin{array}{l}\text { CO-IF-DE- } \\
\mathrm{AO}-\mathrm{CH}\end{array}$ & 5 & 8 & 1 & 1 & 0 & 0 & 0 & 0 & 0 & 1 & 0 & 0 & 0 & 1 & 0 & 1 & 0 \\
\hline $\begin{array}{l}\text { CO-DE-IF- } \\
\mathrm{AO}-\mathrm{CH}\end{array}$ & 7 & 9 & 1 & 1 & 0 & 0 & 0 & 0 & 0 & 1 & 1 & 0 & 0 & 1 & 0 & 1 & 1 \\
\hline $\begin{array}{l}\text { CO-DE-AO- } \\
\text { IF-CH }\end{array}$ & 6 & 6,5 & 1 & 1 & 0 & 0 & 0 & 0 & 1 & 0 & 1 & 0 & 0 & 0 & 0 & 1 & 1 \\
\hline $\begin{array}{l}\text { AO-CO-IF- } \\
\text { DE-CH }\end{array}$ & 5 & 6 & 0 & 0 & 0 & 0 & 0 & 0 & 1 & 1 & 0 & 0 & 1 & 0 & 1 & 0 & 1 \\
\hline $\begin{array}{l}\text { AO-CO-DE- } \\
\text { IF-CH }\end{array}$ & 6 & 6,5 & 0 & 0 & 0 & 0 & 0 & 0 & 1 & 1 & 0 & 0 & 1 & 0 & 1 & 1 & 1 \\
\hline $\begin{array}{l}\text { AO-CO-IF- } \\
\text { CH-DE }\end{array}$ & 6 & 7 & 0 & 0 & 0 & 1 & 0 & 0 & 1 & 1 & 0 & 0 & 1 & 0 & 1 & 0 & 1 \\
\hline $\begin{array}{l}\text { AO-DE-CO- } \\
\text { IF-CH }\end{array}$ & 6 & 6,5 & 0 & 0 & 0 & 0 & 0 & 0 & 1 & 1 & 0 & 0 & 1 & 0 & 1 & 1 & 1 \\
\hline $\begin{array}{l}\text { AO-DE-CH- } \\
\text { IF-CO }\end{array}$ & 9 & 7,5 & 0 & 0 & 1 & 0 & 1 & 1 & 1 & 1 & 0 & 1 & 1 & 0 & 1 & 1 & 0 \\
\hline $\begin{array}{l}\text { AO-CH-IF- } \\
\text { CO-DE }\end{array}$ & 9 & 8 & 0 & 0 & 1 & 1 & 1 & 1 & 1 & 1 & 0 & 1 & 1 & 0 & 1 & 0 & 0 \\
\hline $\begin{array}{l}\mathrm{AO}-\mathrm{CH}-\mathrm{IF}- \\
\mathrm{DE}-\mathrm{CO}\end{array}$ & 8 & 7 & 0 & 0 & 1 & 0 & 1 & 1 & 1 & 1 & 0 & 1 & 1 & 0 & 1 & 0 & 0 \\
\hline $\begin{array}{l}\mathrm{AO}-\mathrm{CH}-\mathrm{DE}- \\
\mathrm{IF}-\mathrm{CO}\end{array}$ & 9 & 7,5 & 0 & 0 & 1 & 0 & 1 & 1 & 1 & 1 & 0 & 1 & 1 & 0 & 1 & 1 & 0 \\
\hline
\end{tabular}




\section{INVESTIGATED OPTICAL DESIGNS}

Since phase B started, in the course of the optical design process, several optical design concepts were investigated in detail and considered for the CFO. In the following a short overview is given on some of these systems (folding and packaging have not been investigated in detail for these systems, so fold mirrors are not shown). Following from the previous sections on requirements and trade-offs, a summary is given on the most critical aspects of the optical design process for the CFO:

- $\quad$ Space envelope for METIS without the LTAO system starts at 750mm downstream from the EELT focal plane. Considering space envelope for the WCU feed it means that the cryostat window shall be located at least 1000mm away from the EELT focal plane. In phase A this distance was $500 \mathrm{~mm}$ and the optical path length increase complicates the optical design, especially, when there is a stringent requirement on the pupil size due to the chopper design.

- The CFO shall be based on a double reimager design. Aiming at an all-spherical design, which naturally means easier manufacturing, verification, alignment and also smaller cost and risk, it is a challenge to achieve the necessary image and pupil quality and at the same time keep the space envelope for the CFO below a reasonable limit.

- The fact that the chopping is done inside the instrument, more specifically in the CFO means that the image quality of the full chopped field using $<5$ " chopping in any direction shall not decrease the overall image quality. Some optical designs cannot satisfy this requirement. One reason is that a sufficiently high level of symmetry is necessary between the optical system before the chopper (collimator) and after the chopper (imager).

- Since the CFO forms the core of the instrument, it has a high number of requirements that it should satisfy. Therefore, up to a certain degree, system level thinking is necessary. Finding the best possible order of the key components was done for this reason and the best solutions naturally also impose additional constraints on the optical design.

\subsection{Spherical mirrors based systems}

Spherical systems, such as the Offner relay (which is the basis of the proposed design) as well as Scwarzschild based systems result easier manufacturing, alignment, integration and testing. These optical designs, through their high level of symmetry, are capable of satisfying the demanding image and pupil requirements and the image quality of the chopped fields can be maintained as well. The centre of curvature of the mirrors are located in the same plane, which also coincides with the plane of the conjugate focal planes (Offner) or the pupil plane (Scwarzschild). These systems offer a high level of flexibility, since they can be combined into double reimagers in various ways. Although they can be re-optimized, they work best, when they are used in 1:1 relays, which means that they require large space envelope and several folding mirrors are needed to reduce the overall dimension of the system built by them (Figure 3). For METIS, which is a cryogenic instrument, optical designs with spherical mirrors are very beneficial because using the aluminium polishing expertise of NOVA, the mirrors can be made of the same material as the mechanical structure and thus any CTE effects (misalignment, stresses, etc.) can be very well mitigated. Built on the experience gained in other instruments developed by the NOVA instrumentation group (VISIR, MIDI, MIRI, X-Shooter, Matisse), a classical Si based aluminium alloy (Al6061, Al6082) with additional hardening (T6) and ageing treatment for long term stability can be a good choice of base material for the cold optical elements and the mechanical structure.
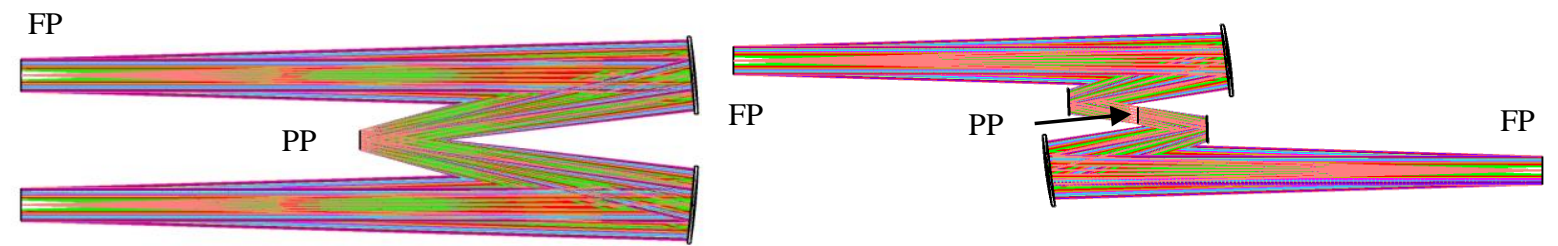

FP

Figure 3 Two all-spherical 1:1 relays, left the Offner, right the Schwarzschild based are shown. They are single reimagers, but they can be combined into double reimagers in various ways. Acronyms: FP: Focal Plane, PP: Pupil Plane. The design proposed for PDR contains Offner type relays. 


\subsection{Aspherical systems with toroidal mirrors and off-axis paraboloids}

Aspherical systems were also investigated. Off-Axis Paraboloid (OAP) mirrors and toroidal mirrors are often used in relay optics, nevertheless they can only provide good image quality in smaller FOV than what is necessary for the CFO. Also, their manufacturing and testing may be challenging due their large size $(\sim 300 \mathrm{~mm})$ and deviation from best fit sphere (larger than $\sim 0.1 \mathrm{~mm}$ ). Their advantage is that less components are needed, since there is generally only one component between the focal and pupil planes. In certain designs there can be even less components, e.g. one can arrive to a very simple design by using only two toroidal mirrors, (left of Figure 4), resulting two consecutive 1:1 relays. Apart from the manufacturing and testing disadvantages mentioned before, it is also very difficult to accommodate a derotator in these designs and the final focal plane usually has very different focal ratio and exit pupil distance compared to what is needed for the subsequent IMG and LMS subsystems. Confocal off-axis paraboloids were also investigated, but designs based on them still could not satisfy the overall image quality and pupil quality requirements up to the degree needed (an example is shown in the right of Figure 4).
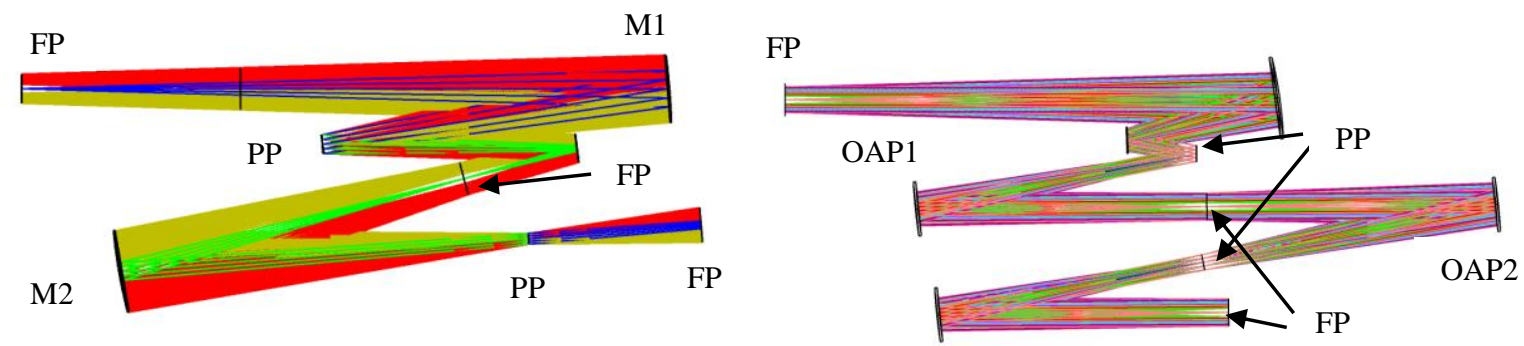

Figure 4 Two aspherical double reimager designs are shown. The system in the left is based on toroidal mirrors (M1 and M2), the one in the right is based on Off-Axis Paraboloids (OAP1 and OAP2). Acronyms: FP: Focal Plane, PP: Pupil Plane.

\subsection{Aspherical systems with three mirror anastigmats}

Three Mirror Anastigmats (TMA-s) are reflective optical systems that typically contains off-axis aspherical components and used extensively in optical designs of ground based and space instrumentation. They are also employed in the optical designs of the LMS and IMG subsystems of METIS. There are various types of them, which can be used in collimators, imagers, relays and afocal systems. For the purpose of the CFO, primarily we looked at relay TMA-s because generally they provide compact solutions with the minimum number of reflections. We investigated relay TMA solutions that provide short back focal length and have an accessible pupil. If two of these TMA-s are combined and their shorter back focal length sides facing towards each other, a very compact solution will result (left in Figure 5). On the other hand, the access to pupils is not very good and although the image and pupil quality are sufficient, they are not as good as in the optical designs of the spherical mirror based designs. Another example is the Cook TMA (right in Figure 5), which inherently has intermediate focal and pupil planes, but its main drawbacks are the optical interface towards the IMG and LMS subsystems and the degraded image quality for chopped fields.
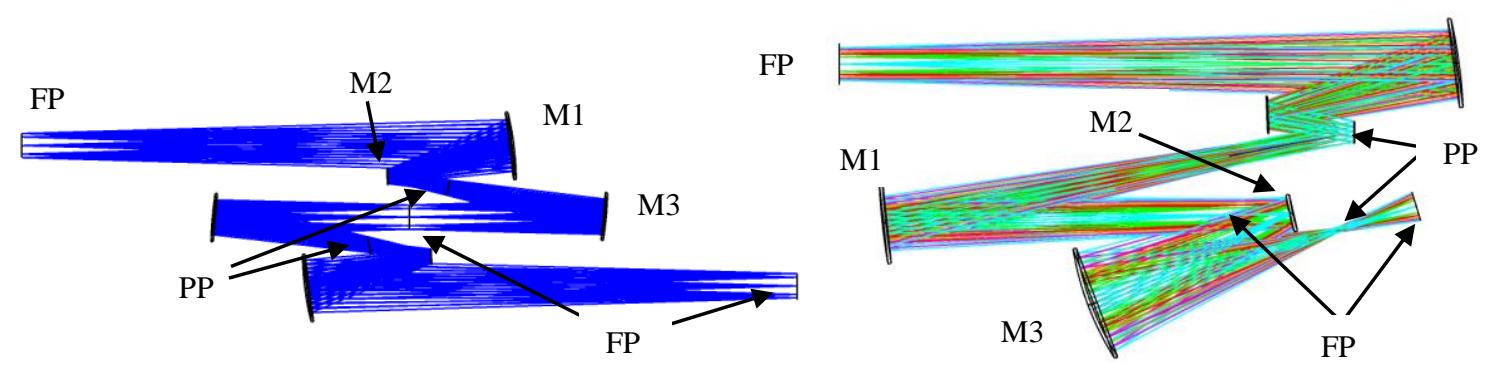

Figure 5 Two examples of TMA based systems are shown. In the left, the same TMA is used twice (M1, M2, M3), in the right the Cook TMA is used (M1, M2, M3).

\subsection{Mixed systems}

Already in some of the examples before, both spherical and aspherical subsystems were used, but the emphasis was on some typical design concept (all-spherical, confocal paraboloids, TMA-s). Here we consider systems, which are more of a mix of various components and optimized altogether in the complete optical path of the CFO. Two examples are shown below (Figure 6), one is based on an Offner relay and two off-axis aspheres (left), the other is based on confocal off-axis 
paraboloids, which are located between two Schwarzschild mirror systems. They both provide very good image and pupil quality and they have a small number of mirrors. Their disadvantage is coming from the fact that they use off-axis aspherical components, which have challenging size $(250 \mathrm{~mm}-300 \mathrm{~mm})$ and departure from best fit sphere (larger than $\sim 0.1 \mathrm{~mm}$ ).
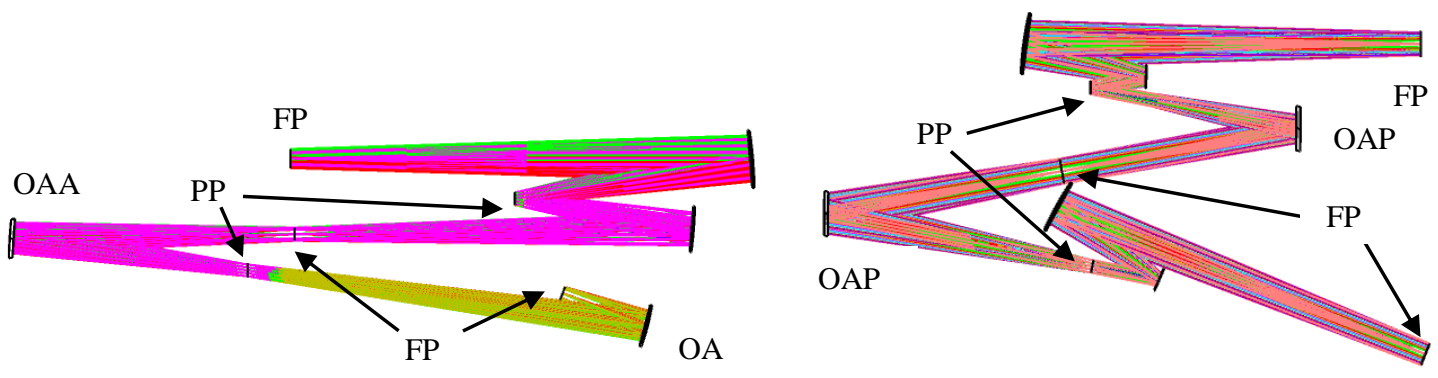

Figure 6 Two examples of mixed systems. One is based on an Offner relay and two off-axis aspheres (left), the other is based on a Schwarzschild and confocal OAP-s (right). Acronyms: FP: Focal Plane, PP: Pupil Plane, OAA: Off-Axis Asphere, OAP: Off-Axis Paraboloid.

\section{PROPOSED PRELIMINARY OPTICAL DESIGN}

We investigated numerous optical design architectures (presented in section 4) and finally we selected an optical design baseline for METIS, which contains only spherical surfaces. The lesson learnt from the investigation was that the additional complications of the apsherical based systems, namely manufacturing, alignment, integration and testing cannot justify the added advantages, which are smaller space envelope and/or improved focal or pupil plane performance. The selected design meet the space envelope requirements by achieving a very efficient folding solution and the focal and pupil plane characteristics of the design are also very good and satisfying the requirements. Additionally, using the aluminium polishing expertise of NOVA for manufacturing the spherical mirrors, the optical surfaces can be made of the same material as the mechanical structure and thus any CTE effects (misalignment, stresses, etc.) can be very well mitigated.

In the following the selected optical design is presented in detail. It is an all-spherical double reimager design based on two 1:1 Offner relays, which are close to telecentric in both conjugates. The classical Offner is a highly symmetrical system with three spherical mirrors (concave-convex-concave) and the pupil is located on the middle convex mirror. A reoptimized Offner design is used in the first reimager and the aim of the re-optimization was to have an accessible pupil (for the cold stop in transmission) instead of having the pupil on the convex second mirror. A classical Offner relay is used in the second reimager, which means that the chopper (located in the pupil) is not flat but it has a spherical surface. The order of key components is the same as the highest ranked configuration in the trade-off study presented in section 0: Cold Stop, Derotator, FP1, AO-pickoff and Chopper. In the following the two reimagers are shown without fold mirrors (Figure 7).

The first reimager includes the derotator, which is located just before FP1. Since the chopper is in the second reimager, it means the full 33" FOV drives the size of the derotator mirrors. The K-mirrors are elliptical in shape and the long axis of the largest mirror reaches $360 \mathrm{~mm}$. Due to the large size of the derotator components, they will not be bare polished Al, but possibly their material will be AlSi, which will be plated with Nickel and post polished to specification.

Other essential CFO component is the chopper. The EELT entrance pupil conjugate has a diameter of $60 \mathrm{~mm}$ on the chopper. The clear aperture of the chopper is not defined yet, it depends on the pupil stability of the EELT, the undersize of the cold stop and the margin that is needed for manufacturing, coating and alignment. Based on the preliminary pupil budget, it is foreseen that it will be between $60-62 \mathrm{~mm}$. The radius of curvature of the chopper is $1098 \mathrm{~mm}$. In phase A the pupil size of the chopper was the same $(60 \mathrm{~mm})$, but in phase A the EELT had a $42 \mathrm{~m}$ diameter entrance pupil, which means that the maximum chop throw is reduced (advantage) and at the same time the chop throw repeatability and stability requirements are reduced (disadvantage). Also, in phase A the chopper clear aperture was $58.2 \mathrm{~mm}$, so it should be slightly increased to accommodate the new $60-62 \mathrm{~mm}$ clear aperture. The larger linear size of the optical surface means that the mechanical system also has to be oversized, which affects power, mass, stiffness and vibrations. Similarly to phase A, all 
three chopper sub-components: mirror, intermediate body and outer ring would be polished spherical (Figure 8), so the spherical surface does not pose any manufacturing challenges. The detailed investigation of a non-flat chopper with $10 \%$ increased size and updated chop-throw characteristics is currently under way.

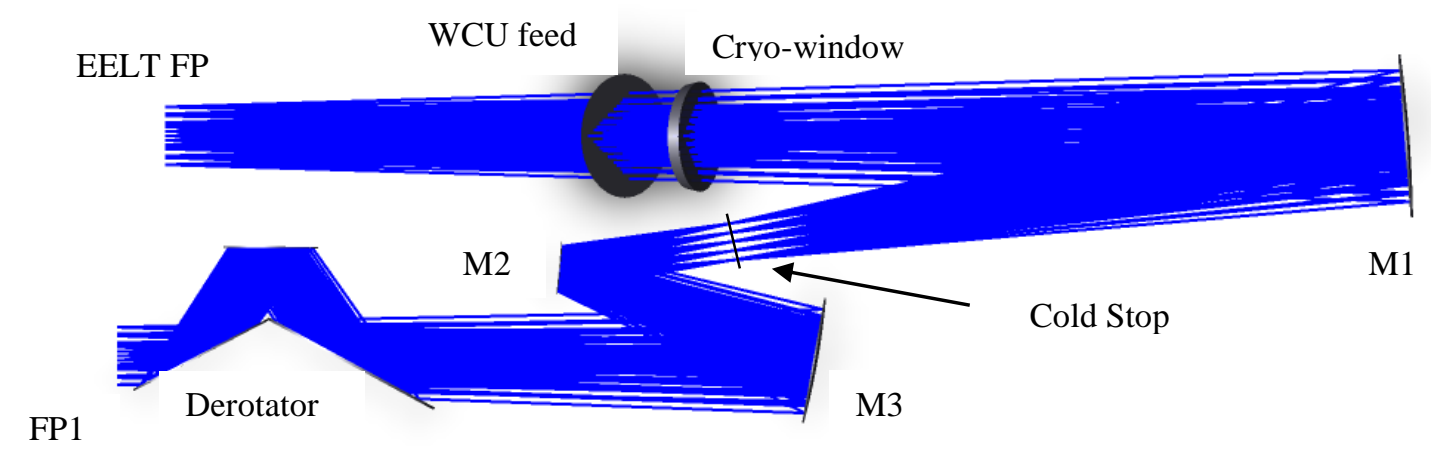

\section{$1 \mathrm{e}+03 \mathrm{~mm}$}

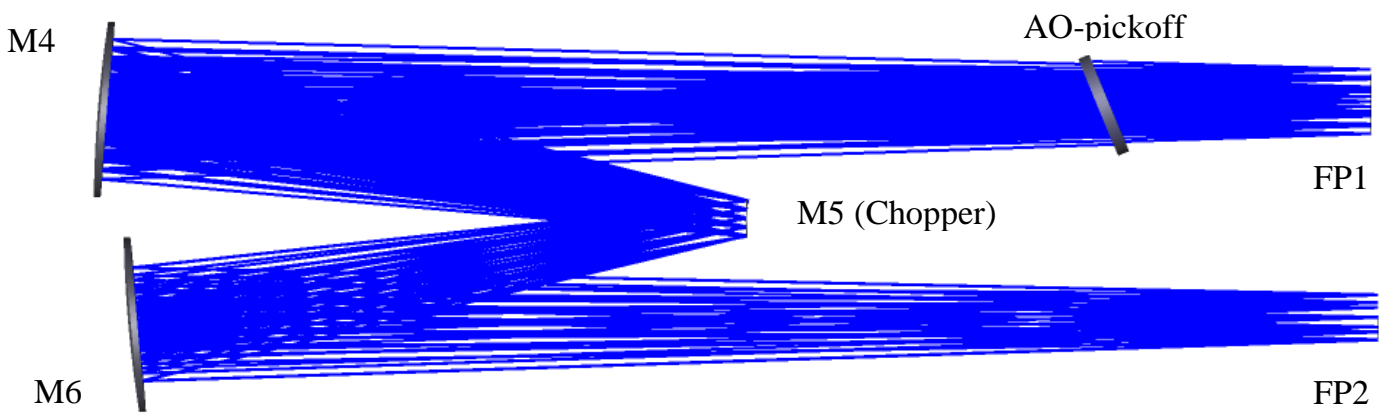

Figure 7 In the top, the layout of the first reimager is shown and the plane, where the three mirrors are located is horizontal. In the bottom, the layout of the second reimager shown; the three mirrors are located in the vertical plane. (FP: Focal Plane).

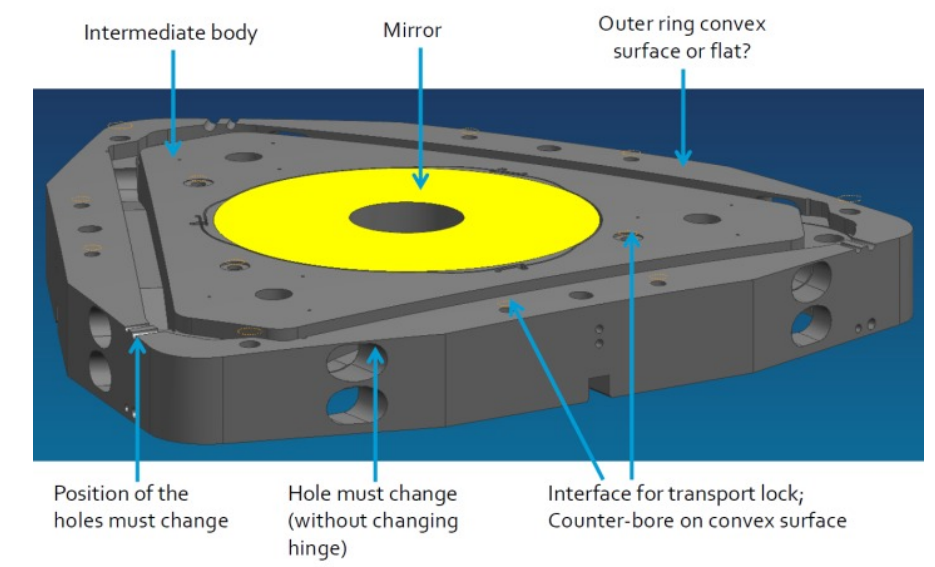

Figure 8 The chopper with spherical mirror surface is shown. The mirror, the intermediate body and the outer ring are polished together, which raises some issues that have to be solved.

In the following the two reimagers are shown together, in Figure 9 without any fold mirrors and in Figure 10 folded. The folding was done in order to keep the instrument in the allocated space envelope, minimize overall volume, position the derotator in a gravity-invariant direction and lower the centre of mass. Also, numerous issues regarding the Cryostat (size, shape, accessibility), the Cold Central Structure (shape, stiffness) and the AIV of the instrument were addressed during the folding process. 


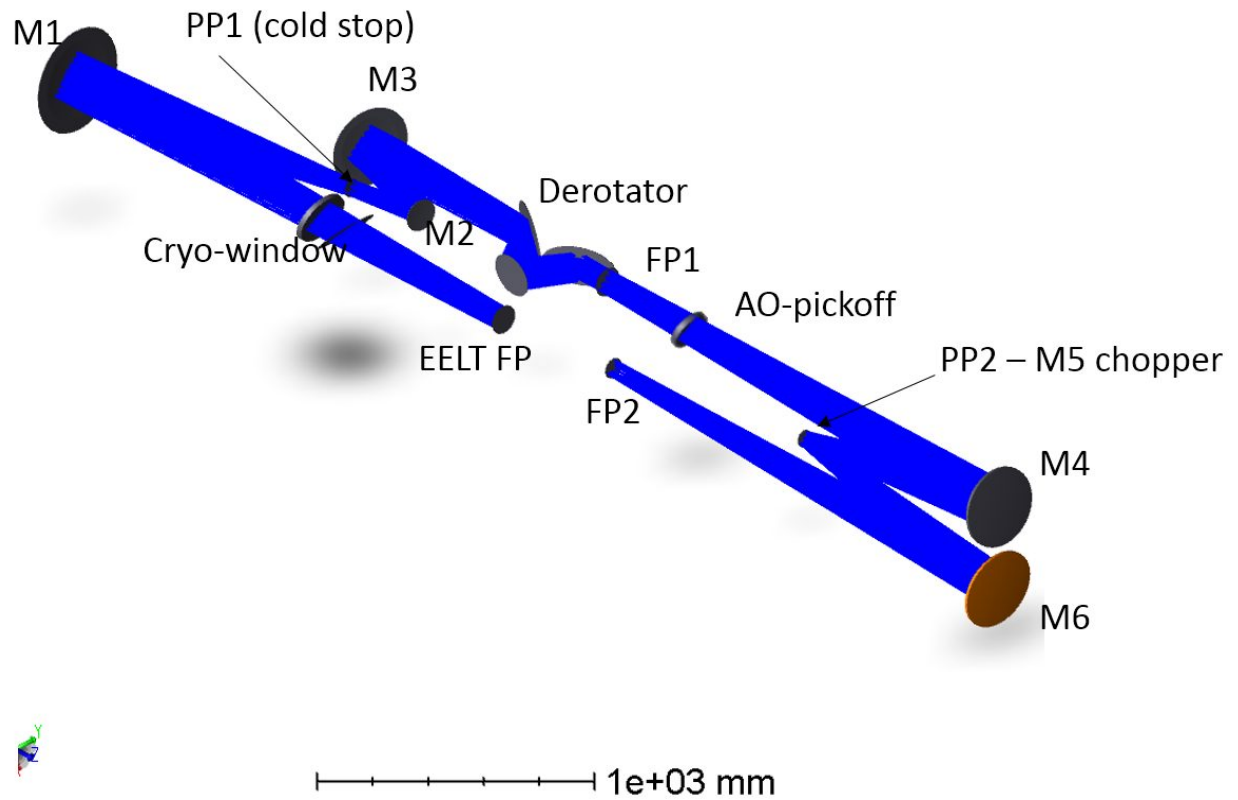

Figure 9 The layout of design 2 is shown without any fold mirrors.

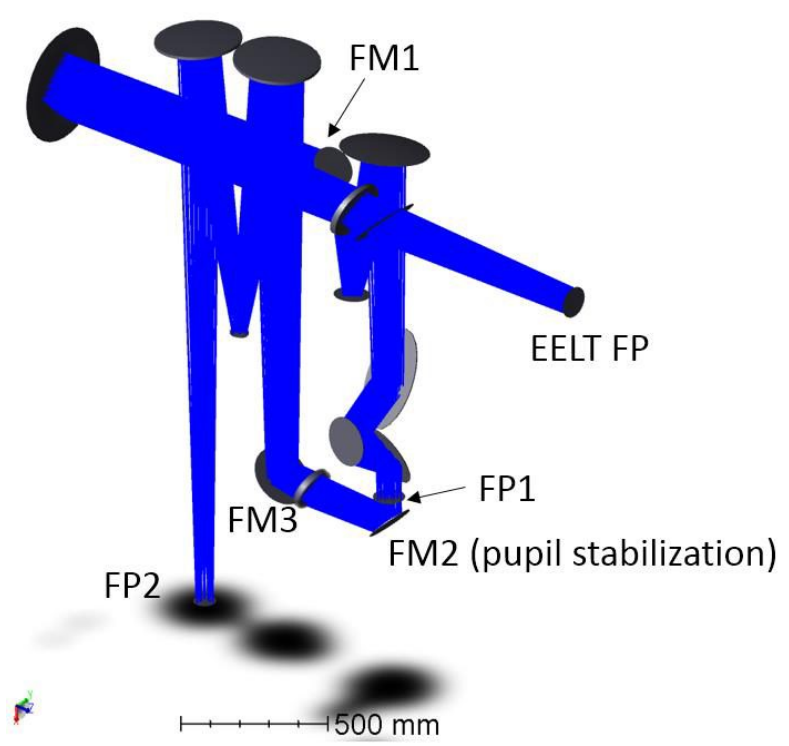

Figure 10 The layout of design 2 is shown with a possible folding solution (fold mirrors labelled as FM1-FM3). The scale and orientation of the incoming beam are the same as in the previous figure.

Due to the fact that the derotator is located just before FP1 (between the two pupils), the two reimagers could not be optimized together to control pupil quality and so the pupil elongation and shear increases or decreases based on the exact position of the derotator. Although the reimagers were optimized independently, image quality is not affected by this and both focal planes provide a Strehl of $>0.99$ at $\lambda=2.9$ micron. In the following sections the image quality and the pupil quality of the design are presented. 


\subsection{Optical characteristics of the focal planes}

In the following table the optical characteristics of the CFO are summarized. The image quality of both focal planes of the $\mathrm{CFO}$ are excellent, they provide $>0.99$ Strehl ratio at all wavelengths in the operational wavelength range of METIS. The focal ratios of FP1 and FP2 are the same and they are equal that of the EELT, which is very beneficial for AIV purposes. Not only the testing and verification of the two reimagers, but the complete CFO and the science subsystems (LMS and IMG) can make use of the same hardware. It is currently investigated, up to what extent the WCU can be used for such purposes.

Table 5 The optical characteristics of the CFO focal planes (FP1 intermediate, FP2 final focal plane).

\begin{tabular}{|c|c|c|c|}
\hline \multicolumn{2}{|c|}{ Parameter } & Value & Note \\
\hline \multicolumn{2}{|c|}{ FOV diameter before chopper } & $33 \operatorname{arcsec}$ & Circular \\
\hline \multicolumn{2}{|c|}{ FOV diameter after chopper } & $23 \operatorname{arcsec}$ & Circular \\
\hline \multirow{5}{*}{ FP1 } & Image quality & Strehl $>0.99$ at $2.9 \mu \mathrm{m}$ for full FOV & \\
\hline & F-ratio & 17.7 & Same as EELT \\
\hline & $\begin{array}{l}\text { Field curvature } \\
\text { (mm at the edge of FOV) }\end{array}$ & $<0.5 \mathrm{~mm}$ & \\
\hline & Distortion (\%) & $<0.05 \%$ & Incl. chopping \\
\hline & Exit pupil distance (m) & $-1451 \mathrm{~m}$ & \\
\hline \multirow{5}{*}{ FP2 } & Image quality & Strehl $>0.99$ at $2.9 \mu \mathrm{m}$ for full FOV & \\
\hline & F-ratio & 17.7 & Same as EELT \\
\hline & $\begin{array}{l}\text { Field curvature } \\
\text { (mm at the edge of FOV) }\end{array}$ & $<0.5 \mathrm{~mm}$ & \\
\hline & Distortion $(\%)$ & $<0.05 \%$ & Incl. chopping \\
\hline & Exit pupil distance (m) & $161 \mathrm{~m}$ & \\
\hline
\end{tabular}

In the following two figures the spot diagrams are shown for FP1 (Figure 11) and FP2 (Figure 12) without chopping ( $1^{\text {st }}$ column) and with the maximum 5" chopping in four directions $\left(2^{\text {nd }}-4^{\text {th }}\right.$ columns). The Airy disk size of the smallest operational wavelength ( 2.9 microns) is also shown.

In Figure 13 the Strehl ratio is shown for FP1 and FP2 for the smallest wavelength of 2.9 microns, for 33" and 23" FOV. In Figure 14 the Strehl ratio is shown for the smallest SCA wavelength (1.5 micron) for FP1 (FOV is 33"). It shows the image quality of the CFO just before light enters into the SCA.

Finally, the Strehl ratios are depicted, when the maximum 5" chopping is done in four different directions for FP1 and FP2 for 23" FOV (Figure 15). 


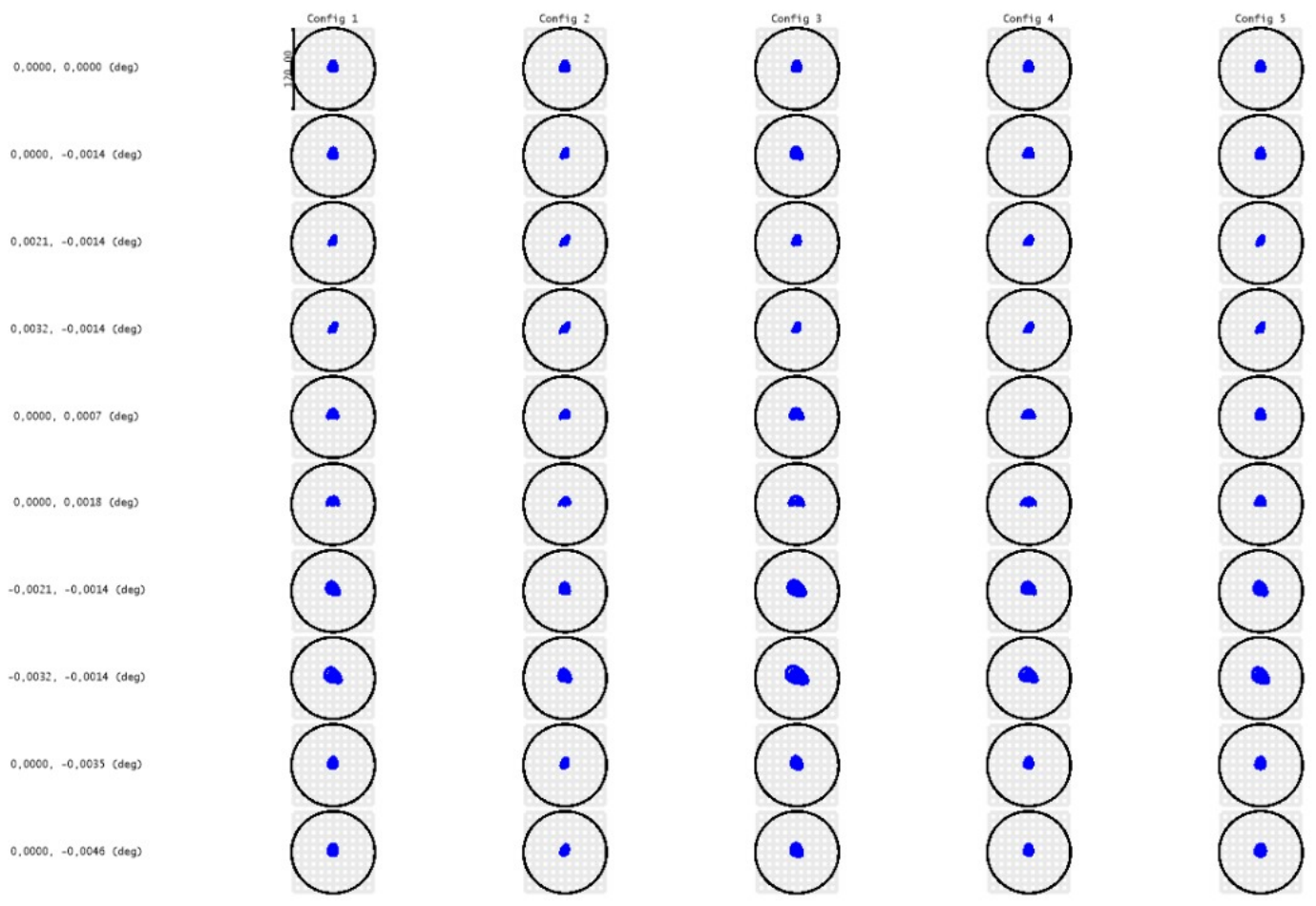

Figure 11 The spot diagrams at FP1 of the CFO for the various field positions (rows) and for the different chopping directions (column) are shown. No chopping ( $1^{\text {st }}$ column $),+-5^{\text {" }}$ in $x\left(2^{\text {nd }} / 3^{\text {rd }}\right.$ columns $)$ and $+/-5^{\text {" }}$ in y $\left(4^{\text {th }} / 5^{\text {th }}\right.$ columns $)$. The airy disk diameter for the 2.9 micron wavelength is also shown.
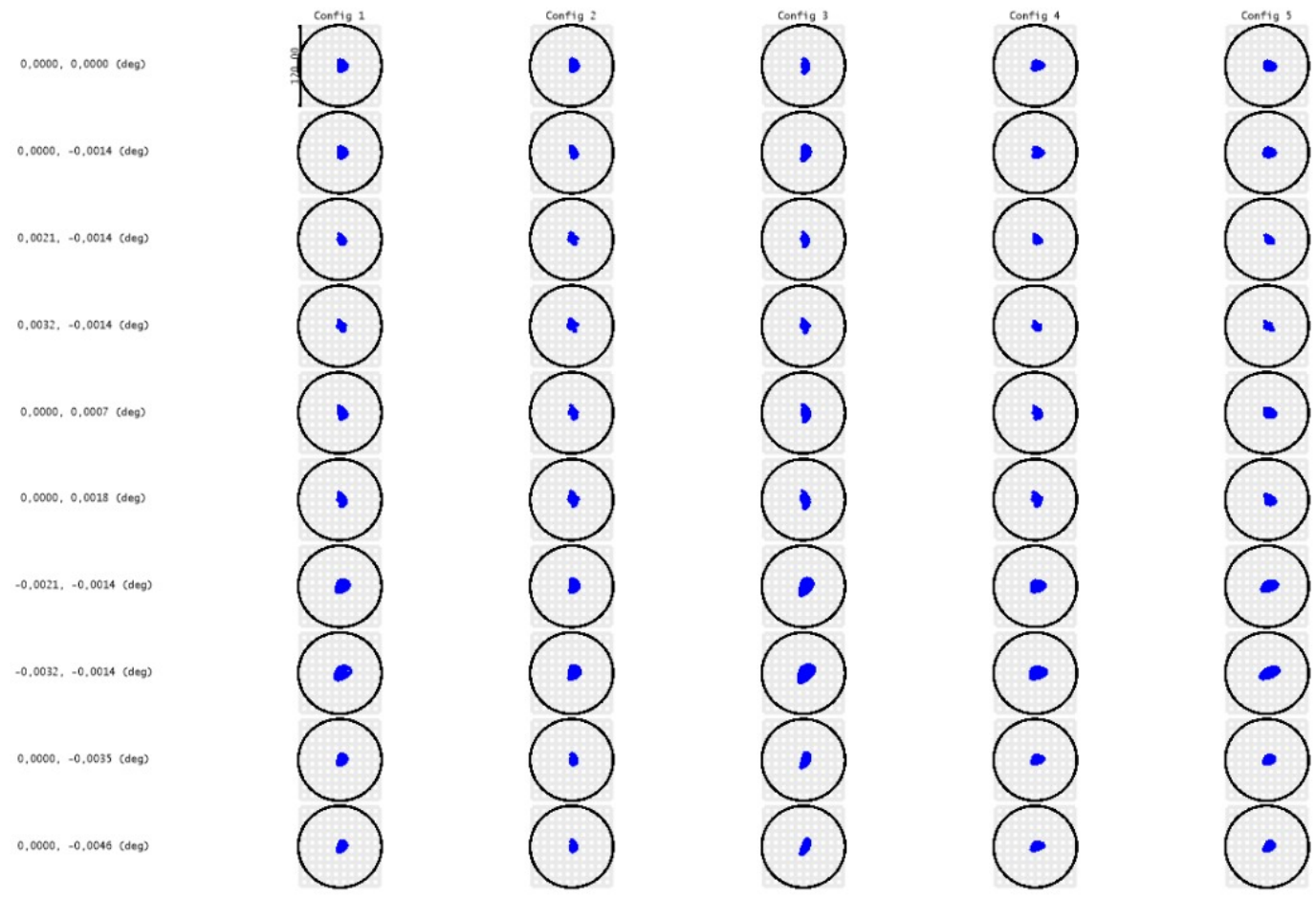

Figure 12 The same as above, but for FP2. 

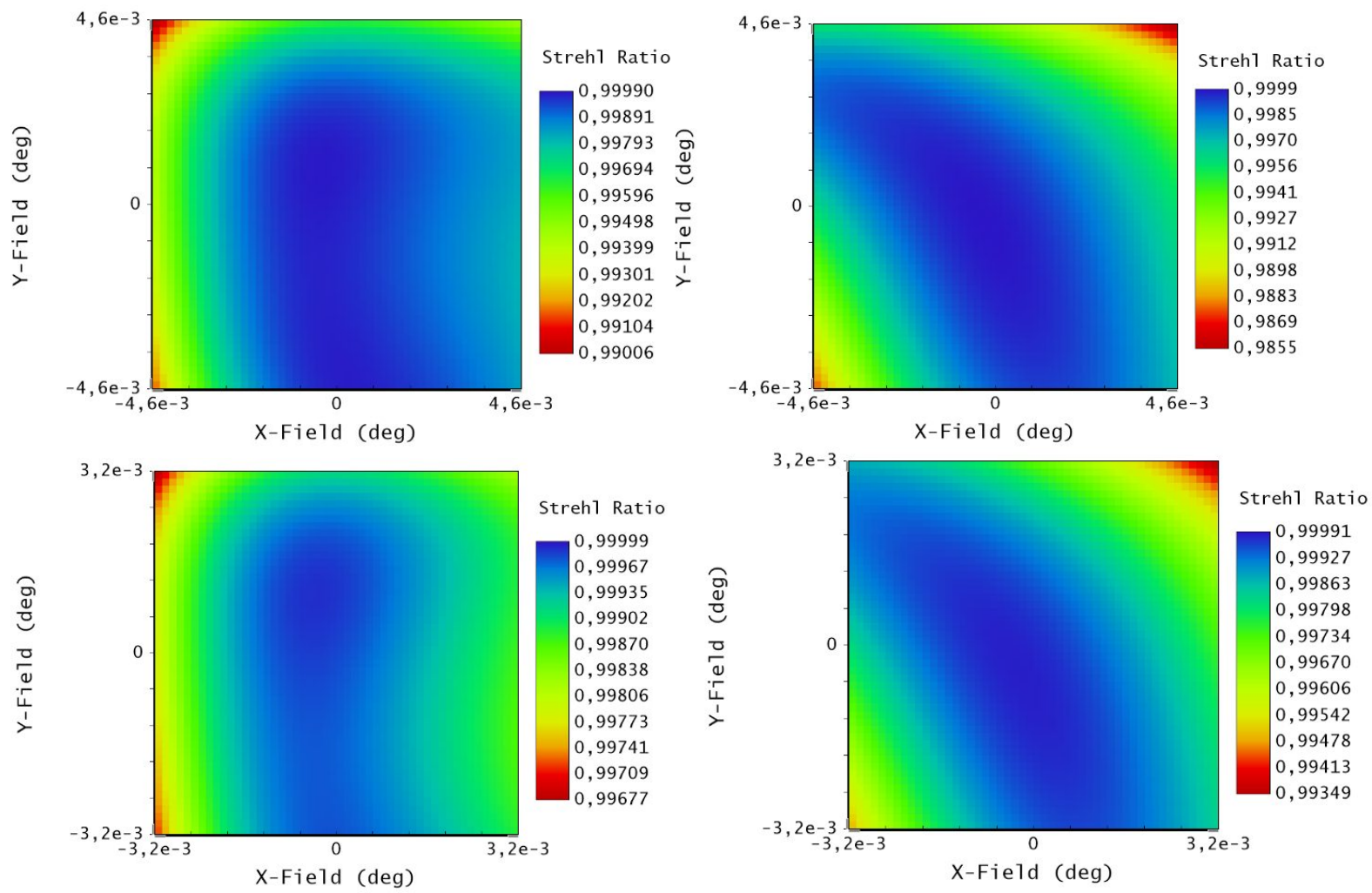

Figure 13 The Strehl ratios are shown for FP1 (left) and FP2 (right) for 2.9 micron wavelength. The side of the shown square FOV is 33 " (top row) and 23" (bottom row).

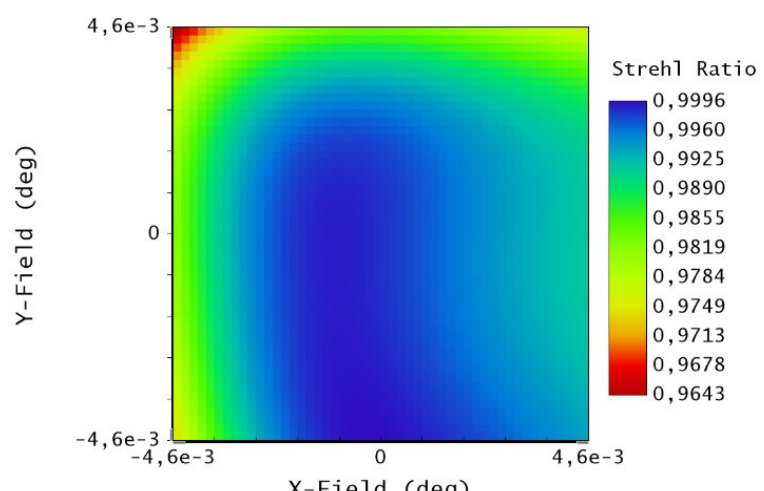

Figure 14 The Strehl ratio is shown for the smallest SCA wavelength (1.5 micron) for FP1. The side of the shown square FOV is 33 ". It shows the image quality of the CFO just before it enters into the SCA. 

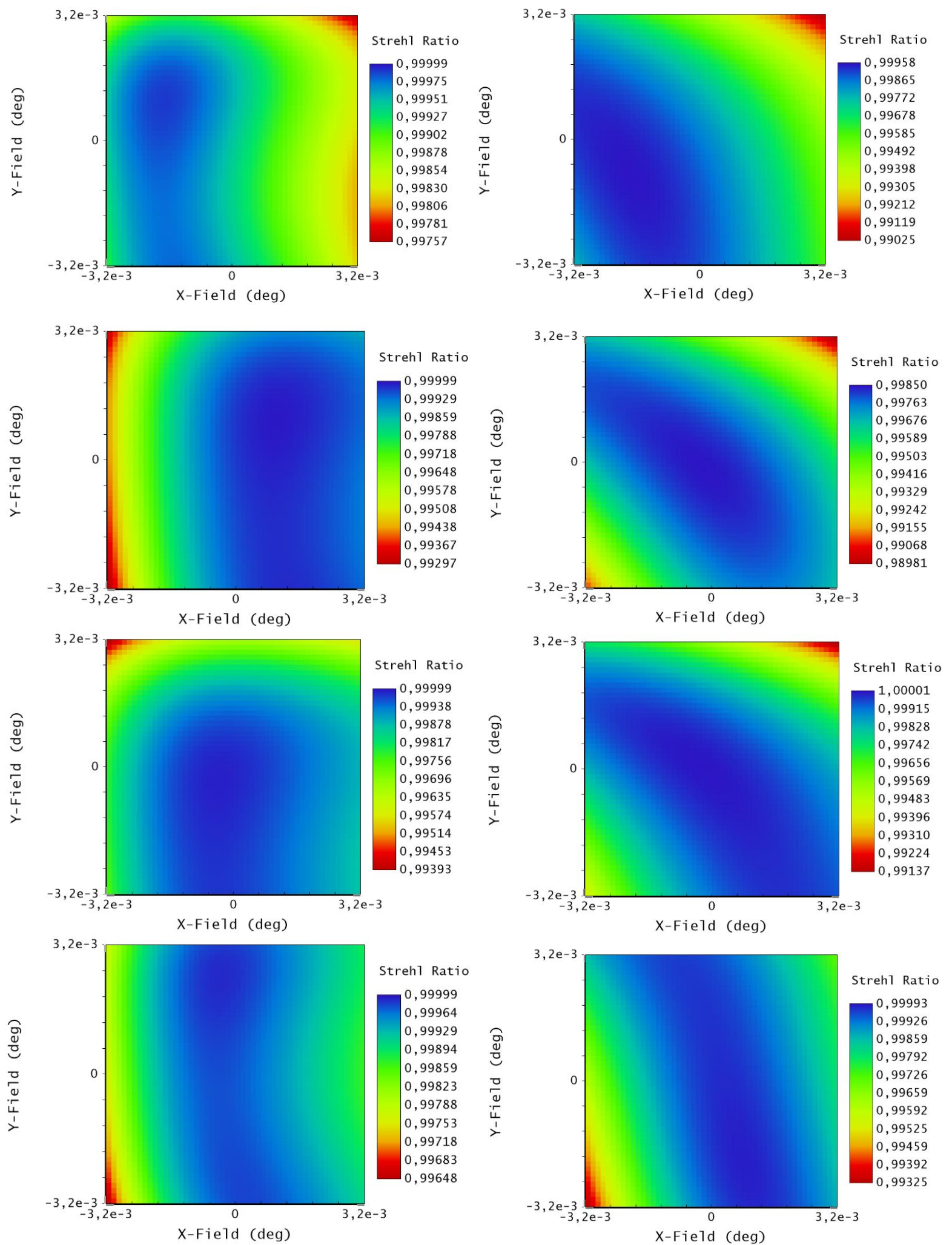

Figure 15 The Strehl ratios are shown for FP1 (left column) and FP2 (right column) for 2.9 wavelength for the chopped fields. $+/-5$ " in $x\left(1^{\text {st }} / 2^{\text {nd }}\right.$ row $)$ and $+/-5$ " in y $\left(3^{\text {rd }} / 4^{\text {th }}\right.$ row $)$. The side of the shown square field of view is 23 ". 


\subsection{Optical characteristics of the pupil planes}

In the following table the optical characteristics of the pupil planes of the CFO are shown. In Figure 16 the two pupils are shown for non-chopped and 5" chopped FOV (in four different directions). The depicted pupil diameter corresponds to the EELT entrance pupil diameter.

Table 6 The optical characteristics of the CFO pupil planes (PP1 chopper, PP2 cold stop pupil planes).

\begin{tabular}{|c|c|c|c|}
\hline \multicolumn{2}{|l|}{ Parameter } & \multirow{2}{*}{$\begin{array}{l}\text { Value } \\
70 \mathrm{~mm}\end{array}$} & \multirow{2}{*}{$\begin{array}{l}\text { Note } \\
\text { Conj. to EELT EP }(38542 \mathrm{~mm})\end{array}$} \\
\hline \multirow{6}{*}{$\begin{array}{l}\text { PP1 (Cold Stop + HCI masks) in } \\
\text { transmission }\end{array}$} & Pupil diameter & & \\
\hline & Size of cold stop conjugate & $65.3 \mathrm{~mm}$ & $\begin{array}{l}2.5 \% \text { undersized cold stop wrt 'all } \\
\text { glass' diameter }\end{array}$ \\
\hline & Pupil shear & $<0.4 \%$ & For 33" FOV \\
\hline & Pupil elongation & $<1.0 \%$ & For 33" FOV \\
\hline & Combined pupil errors & $<1.5 \%$ & Shear, elongation, chopping \\
\hline & $\begin{array}{l}\text { Radius of curvature of } \\
\text { chopper mirror }\end{array}$ & $1098 \mathrm{~mm}$ & Non-flat chopper. \\
\hline \multirow{5}{*}{ PP2 (Chopper) in reflection } & Pupil diameter & $60 \mathrm{~mm}$ & Conj. to EELT EP (38542mm) \\
\hline & Undersized dimension & $56 \mathrm{~mm}$ & $\begin{array}{l}2.5 \% \text { undersize wrt 'all-glass' } \\
\text { diameter }\end{array}$ \\
\hline & Pupil shear & $<1.5 \%$ & For 23" FOV \\
\hline & Pupil elongation & $<3.3 \%$ & For 23" FOV \\
\hline & Combined pupil errors & $<5.0 \%$ & Shear, elongation, chopping \\
\hline
\end{tabular}
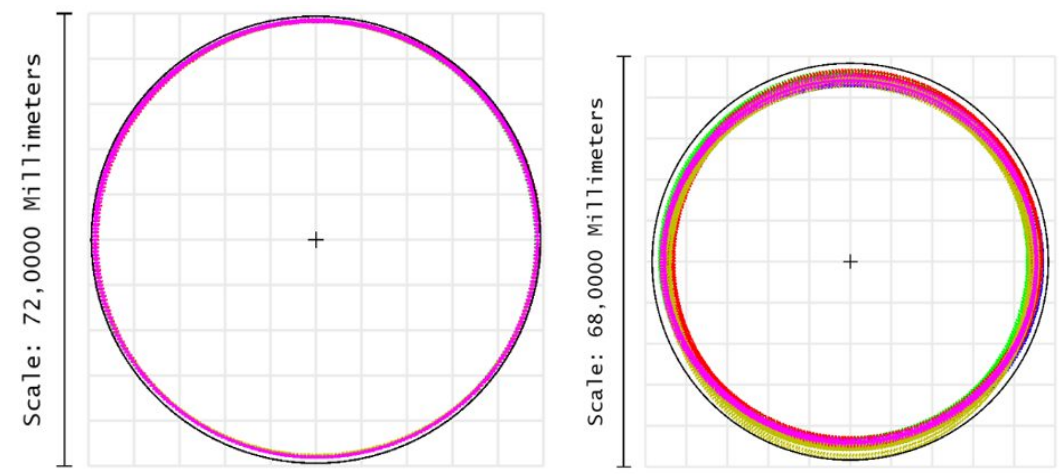

Figure 16 PP1 (left) and PP2 (right), pupils of the CFO are shown. Non-chopped and 5" chopped (in four different directions) FOV also sown. In the worst case (derotator position) the combined pupil errors can reach 5\%. 


\section{CONCLUSIONS}

We presented the requirements and detailed the most critical ones that drive the design of the CFO. Based on a dedicated numerical scoring system, we determined the highest ranked design configuration with respect to the order of the key components in the double reimager based optical system: Cold Stop, Derotator, FP1, AO-pickoff and Chopper. We investigated numerous optical architectures (spherical and aspherical) and presented the selected CFO design.

The selected optical design is an all-spherical system and the two reimagers are both 1:1 relays. The first reimager is a reoptimized Offner system with an accessible pupil in between the second and third mirrors, where the cold stop is located. The derotator is positioned just before the intermediate focal plane (FP1). The second reimager is a classical Offner relay, it contains the AO pick-off and subsequently the chopper (convex mirror) in the pupil. We presented the excellent image quality (spot sizes and Strehl ratio) and pupil plane characteristics of the design. We showed a very convenient folding solution, which minimizes overall volume, position the derotator in a gravity-invariant direction and lower the centre of mass among other advantages.

\section{REFERENCES}

[1] Brandl, B. R. et al., "Status of the mid-infrared E-ELT imager and spectrograph METIS," Proc. SPIE 9908-74 (2016).

[2] Kenworthy, M. et al., "High-contrast imaging with METIS" Proc. SPIE 9908-378 (2016). 